\title{
Numerical Simulation of a Turbulent Magnetic Dynamo
}

\author{
S. Kenjereš and K. Hanjalić \\ Department of Multi Scale Physics and J.M. Burgerscentre for Fluid Dynamics, Delft University of Technology, \\ Lorentzweg 1, 2628 CJ Delft, The Netherlands
}

(Received 26 June 2006; published 6 March 2007)

\begin{abstract}
We present numerical simulations of a turbulent magnetic dynamo mimicking closely the Riga-dynamo experiment at $\mathrm{Re} \approx 3.5 \times 10^{6}$ and $15 \leq \mathrm{Re}_{m} \leq 20$. The Reynolds-averaged Navier-Stokes equations for the fluid flow and turbulence field are solved simultaneously with the direct numerical solution of the magnetic field equations. The fully integrated two-way-coupled simulations reproduced all features of the magnetic self-excitation detected by the Riga experiment, with frequencies and amplitudes of the selfgenerated magnetic field in good agreement with the experimental records, and provided full insight into the unsteady magnetic and velocity fields and the mechanisms of the dynamo action.
\end{abstract}

DOI: 10.1103/PhysRevLett.98.104501

Complex interactions between turbulent flow of electrically conductive fluids and electromagnetic fields play the key role in many physical phenomena in nature and technology. Of particular interest is the conversion of the mechanical energy of a moving electrically conductive medium into the magnetic energy, known as the magnetic dynamo. It is believed that the magnetic dynamo effects are responsible for the creation of magnetic fields in spiral galaxies, stars, and planets (including Earth's magnetic field), e.g., [1]. In order to create the favorable conditions for possible self-excitation of a magnetic field, it is necessary to achieve regimes where stretching of the magnetic field will overcome its resistive damping. This condition is defined by the critical magnetic Reynolds number $\mathrm{Re}_{m}=$ $U L / \lambda>1$ (where $U$ and $L$ are typical velocity and length scale, respectively, and $\lambda$ is magnetic diffusivity). In order to reach such conditions, one needs relatively large length scales and high velocities - even for the best electrically conductive fluids (e.g., liquid sodium, $\lambda \approx 1 / \mu_{0} \sigma=$ $0.1 \mathrm{~m}^{2} / \mathrm{s}$ ), where $\mu_{0}$ and $\sigma$ are the magnetic permeability and electric conductivity. For such configurations, the hydrodynamical Reynolds number $(\operatorname{Re}=U L / \nu)$ will be also very high $\left(\operatorname{Re} \approx 10^{5}-10^{6}\right)$, making the flow highly turbulent. Experimental studies of magnetic fluid dynamos face many practical problems associated with large dimensions of setups and potentially hazardous working fluids (sodium). This explains why it was not until late 1999 when two experimental groups in Riga ([2-6]) and Karlsruhe ([7-9]) finally and independently succeeded in detecting the self-excitation and subsequent sustenance of a magnetic field for the very first time. Although this is an important step towards understanding and explaining the Earth's magnetic field from the magnetic dynamo effect, both experiments were not designed to actually mimic the Geo-Dynamo (Earth-like) conditions, but rather to provide experimental proof of the magnetic field self-amplification.

Despite their remarkable success, the experimental studies provided only the time records of the magnetic field components at particular locations - so information addressing the detailed spatial distribution of the magnetic
PACS numbers: 47.11.- j, 47.27.E-, 47.65.-d, 91.25.Cw

field and its dynamics is still missing. Numerical simulation, if proven reliable, is the only way to provide detailed information on the complex physics of the time-dependent fluid flow, turbulence, and electromagnetic fields and their interactions. In this Letter, we present numerical simulations of the dynamo action in the Riga-dynamo experimental setup, Fig. 1. The characteristic hydrodynamical Re is very high $\left(\operatorname{Re}=3.5 \times 10^{6}\right)$ and the magnetic $\operatorname{Re}_{m}(15 \leq$ $\mathrm{Re}_{m} \leq 20$ ) should be sufficient to potentially trigger a selfexcitation of the magnetic field. For these regimes, the magnetic Prandtl number is very low, $\operatorname{Pr}_{m}=\nu / \lambda=$ $\left(\eta_{u} / \eta_{B}\right)^{4 / 3}=6.5 \times 10^{-6}$, implying that the magnetic diffusive length scale $\eta_{B}=\left(\lambda^{3} / \varepsilon\right)^{1 / 4}$ is much larger than the velocity viscous scale $\eta_{u}=\left(\nu^{3} / \varepsilon\right)^{1 / 4}$.

The governing momentum and magnetic induction equations (for incompressible fluid) are

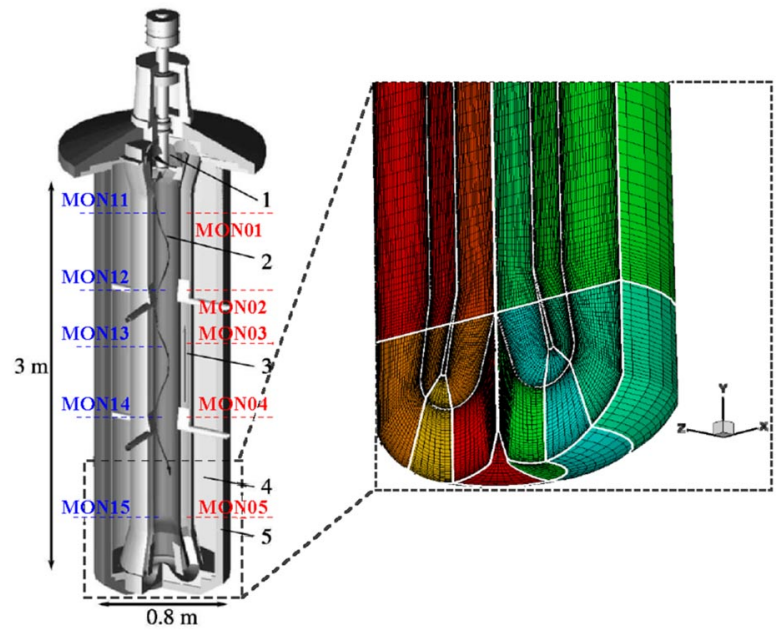

FIG. 1 (color online). The Riga-dynamo experimental setup [3]: 1-propeller; 2-inner cylinder with strong helical flow; 3outer passage with back flow; 4-surrounding ring of sodium initially at rest; 5-thermal insulation; Below-a 3D view of a part of the numerical mesh used for simulations. 


$$
\begin{aligned}
& \frac{\partial U_{i}}{\partial t}+U_{j} \frac{\partial U_{i}}{\partial x_{j}}=\frac{\partial}{\partial x_{j}}\left[\nu\left(\frac{\partial U_{i}}{\partial x_{j}}+\frac{\partial U_{j}}{\partial x_{i}}\right)\right]-\frac{1}{\rho} \frac{\partial p}{\partial x_{i}} \\
& +\underbrace{\frac{1}{\rho \mu_{0}}\left(B_{k} \frac{\partial B_{i}}{\partial x_{k}}-B_{k} \frac{\partial B_{k}}{\partial x_{i}}\right)} \\
& \mathbf{F}^{\mathbf{L}}=\mathbf{1} / \mu_{\mathbf{0}}(\nabla \times \mathbf{B}) \times \mathbf{B} \\
& \frac{\partial B_{i}}{\partial t}+U_{j} \frac{\partial B_{i}}{\partial x_{j}}=\frac{\partial}{\partial x_{j}}\left(\frac{1}{\mu_{0} \sigma} \frac{\partial B_{i}}{\partial x_{j}}\right)+B_{j} \frac{\partial U_{i}}{\partial x_{j}}
\end{aligned}
$$

with the solenoidal conditions $\partial U_{i} / \partial x_{i}=0$ and $\partial B_{i} / \partial x_{i}=0$. The most exact numerical approach is to directly solve these equations in space and time (direct numerical simulation, DNS) but due to formidable computational requirements, this approach can be applied only to flows in relatively simple geometries and for low Re number, [10-13]. In order to overcome these limitations, the numerical schemes using hyperviscosity and hyperdiffusivity have been introduced (aimed at extending the inertial subrange and consequently to reduce the dissipative subrange) [14], but such approaches provide no account for turbulence, which strongly affects the velocity field and its interaction with the magnetic field.

Here, we introduce a hybrid approach where the velocity and turbulence fields are solved by the transient ReynoldsAveraged Navier-Stokes (RANS) method and the electromagnetic variables are resolved directly (DNS). Such a combined method, as well as the use of the RANS approach for treating the velocity field and turbulence properties (which is the only approximation involved in the simulations), is regarded as justified because of the mentioned disparity in scales, characterized by the low $\operatorname{Pr}_{m}$. A similar hybrid merging is applied in [11] where the Lagrangian-averaged MHD equations are introduced (albeit for relatively simple Taylor-Green vortex and $\mathrm{Re} \leq$ $\left.1.5 \times 10^{4}\right)$. In the RANS approach, the instantaneous fields are decomposed into their time- or ensemble-averaged values and the fluctuations, i.e., $U_{i}=\bar{U}_{i}+u_{i}, B_{i}=\bar{B}_{i}+$ $b_{i}$. As a result of this averaging, additional unknown correlations appear, $\overline{u_{i} u_{j}}, \overline{b_{i} b_{j}}, \overline{u_{i} b_{j}}$, etc., [15-19]. In order to close this system, additional equations for these correlations must be introduced, what constitutes a turbulence closure model. The starting point in our analysis is the second-moment (Reynolds stress) $\overline{u_{i} u_{j}}-\varepsilon$ model with the newly included additional MHD effects (the source/sink and the redistributive terms are taken into account), [17], which was validated in a range of generic situations for $5 \times 10^{3} \leq \mathrm{Re} \leq 10^{5}$ and $0 \leq \mathrm{Ha} \leq 1000$-where $\mathrm{Ha}=$ $B_{0} L \sqrt{\sigma / \rho \nu}$ is Hartmann number) showing in all cases good agreement with the available experiments, DNS and large eddy simulations (LES) studies, [17]. For the high Re Riga-dynamo with the specific solid-body-like strong initial swirl, we opted for a simplified model and solved the transport equation for the turbulence kinetic energy $k=$ $0.5 \overline{u_{i} u_{i}}$ instead of for all six turbulent-stress components $\overline{u_{i} u_{j}}$. A justification came from our prior simulations of the 1:2 scale-down water experimental setup (for which de- tailed laser Doppler anemometry (LDA) measurements are available), which demonstrated good agreement between the full second-moment closure and its simplified twoequation $k-\varepsilon$ variant. In this model, we have $\overline{u_{i} u_{j}}=$ $2 / 3 k \delta_{i j}-\nu_{t}\left(\partial U_{i} / \partial x_{j}+\partial U_{j} / \partial x_{i}\right)$, where the turbulent viscosity is calculated as $\nu_{t}=c_{\mu} k^{2} / \varepsilon$ and additional turbulent "MHD" contributions in $k$ and $\varepsilon$ are modeled as $S_{k}^{M}=-\sigma / \rho B_{0}^{2} k \exp \left(-C_{1}^{M} \sigma / \rho B_{0}^{2} k / \varepsilon\right), S_{\varepsilon}^{M}=S_{k}^{M} \varepsilon / k$, respectively $\left(B_{0}=\sqrt{B_{x}^{2}+B_{y}^{2}+B_{z}^{2}}\right)$. This simplified version of the model given in [16,20] has been extensively validated and applied to a variety of flows such as flow and turbulence reorganization in turbulent thermal convection subjected to external magnetic fields of different orientations and strength [21], turbulent magneto-aerodynamics [22], free surface flows in fusion engineering [23], solar wind turbulence [24], etc.

The closed system of equations $U_{i}-B_{i}-k-\varepsilon$ is discretized by a finite-volume integrated solver for the structured multiblock nonorthogonal geometries with a local (block specified) grid refinement. The diffusive terms are disretized by the second-order central difference scheme (CDS). The monotonicity preserving total variation diminishing (TVD) scheme is used for the convective terms. The time integration is performed by a fully implicit secondorder scheme with three-consecutive time steps. Typical time-step values are specified to be 1/100 from the experimentally observed periodic time intervals of the selfexcited magnetic field components, i.e., $\Delta t=1 / 100 \mathrm{~s}$. The numerical grid distribution used for simulations is shown in Fig. 1. Note that the complete geometry of the experimental configuration is generated (including inner cylinder, outer annular passage, and surrounding ring with sodium initially at rest) since it is necessary to solve the magnetic induction equation over the entire domain - not only in domains with active fluid motion. In total, 80 structured blocks are used with approximately $4 \times 10^{6}$ control volumes. The parallel code execution with 64 CPUs resulted in almost ideal load balancing between processors at the SGI Origin 3800 system with 1024 CPUs [25].

In simulations, the swirl generation is mimicked by imposing the experimentally determined axial and tangential velocity components in the propeller exit plane. Because of the highly turbulent flow regime, the wall functions are used for providing the wall boundary conditions for hydrodynamical variables. The vertical magnetic field boundary condition is imposed for the magnetic field components at the outer side of the surrounding ring of the sodium initially at rest, [6,26]. The very first step in numerical simulation was to obtain the fully developed (statistically steady) RANS solutions for the fluid flow and turbulence field (without electromagnetic effects). Then, the electromagnetic field is activated and the timedependent integration is performed. Through the earlier extensive testing of the solver in the kinematic mode (with no backreaction of the generated magnetic field on the fluid 
flow) it was proved that the vertical magnetic field condition $([6,26])$ provided reasonable predictions of the theoretically estimated (based on the convective instabilities of the Ponomarenko dynamo, [4,6], $\operatorname{Re}_{m}^{c}=17.7$ ) critical thresholds, $15 \leq R e_{m}^{c} \leq 20$.

The numerically obtained frequencies of the axial component of the self-induced magnetic field as a function of the rotation rates, Fig. 2, show good agreement with experiments. The next step was to activate the two-way coupling, i.e., to simultaneously solve the entire system with the backreaction of the Lorentz force on both the momentum $\left(\bar{U}_{i}\right)$ and turbulence parameters equations $(k, \varepsilon)$. After the initial exponential growth of the selfexcited magnetic field in the kinematic regime, the Lorentz force increases to the level which is strong enough to significantly affect the underlying fluid flow. Essentially, the Lorentz force begins to brake the fluid flow causing the significant reduction in the magnetic field growth rate. Now, through the backreaction mechanism, the original intensity of the Lorentz force will be reduced, creating again favorable conditions for the increase in the magnetic field. This balance is characterized by the eventual zerogrowth rate of the induced magnetic field when the system reaches the saturation regime. The effects of the two-way coupling between the fluid flow and electromagnetic field can be viewed in Fig. 3 where the situations with and without backreaction have been considered in parallel (under identical initial flow and electromagnetic condition, defined by $\operatorname{Re}=3.5 \times 10^{6}, \operatorname{Re}_{m}=18$ ). For the case without backreaction, magnetic field components exhibit an exponential growth (Fig. 3-top and bottom left) - as expected. The backreaction mechanism reduced this exponential growth, and the saturation regime is finally achieved. The simulations show the strongest amplification of the axial magnetic field component $(--)$ in the middle part of the setup (MON2, $B_{y}$ ) as observed in [3], confirming a strong criticality dependence on the underlying fluid flow and turbulence conditions, Fig. 3-middle. The direct comparison of the time-recorded experimental and numerical axial magnetic field signal $\left(B_{y}\right)$ at the middle height of the outer passage is shown in Fig. 3-bottom right. It can be concluded that the numerical simulations predicted amplitudes of the axial magnetic field component in very good agreement with experiments, while the frequency is slightly underpredicted. The detailed information about

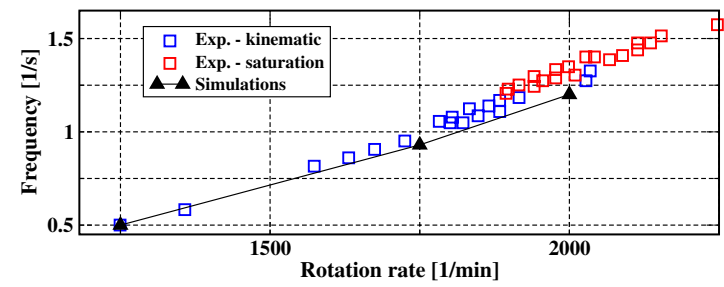

FIG. 2 (color online). Frequencies of the self-induced magnetic field as a function of the rotational rates: comparison with experiments in kinematic and saturation regimes. Experimental data by Gailitis . the 4-dimensional (spatial-temporal) variations of the turbulence statistics, velocity, turbulence, and magnetic field - generated by the simulations - makes it possible to go much further in analyzing the complex interactions and the mechanisms of a turbulent magnetic dynamo. In order to provide insights into the dynamo core turbulence (still inaccessible to measurements), the time evolutions of the turbulent kinetic energy $\left(\mathrm{TKE}=0.5 \bar{u}_{i}^{2}\right)$ at characteristic locations in the inner cylinder and the outer passage are shown in Fig. 4. The initial state corresponds to the fully developed steady turbulent flow conditions prior to the activation of the magnetic induction equations. The relatively short initial period $(0-2 \mathrm{sec})$ is characterized by the low-magnitude Lorentz force and weak or totally absent disturbances of the TKE. Then, after reaching saturation levels of the Lorentz force, the period with intensive disturbances occurs $(>3 \mathrm{sec})$. In this stage, an interesting flow and turbulence reorganization takes place. The selfgenerated Lorentz force significantly suppresses intensity of turbulence in the inner cylinder with strongest suppression in its lower part. The TKE in the outer passage is initially suppressed too, but after a short recovery period, its intensity is increased due to the fading of the Lorentz
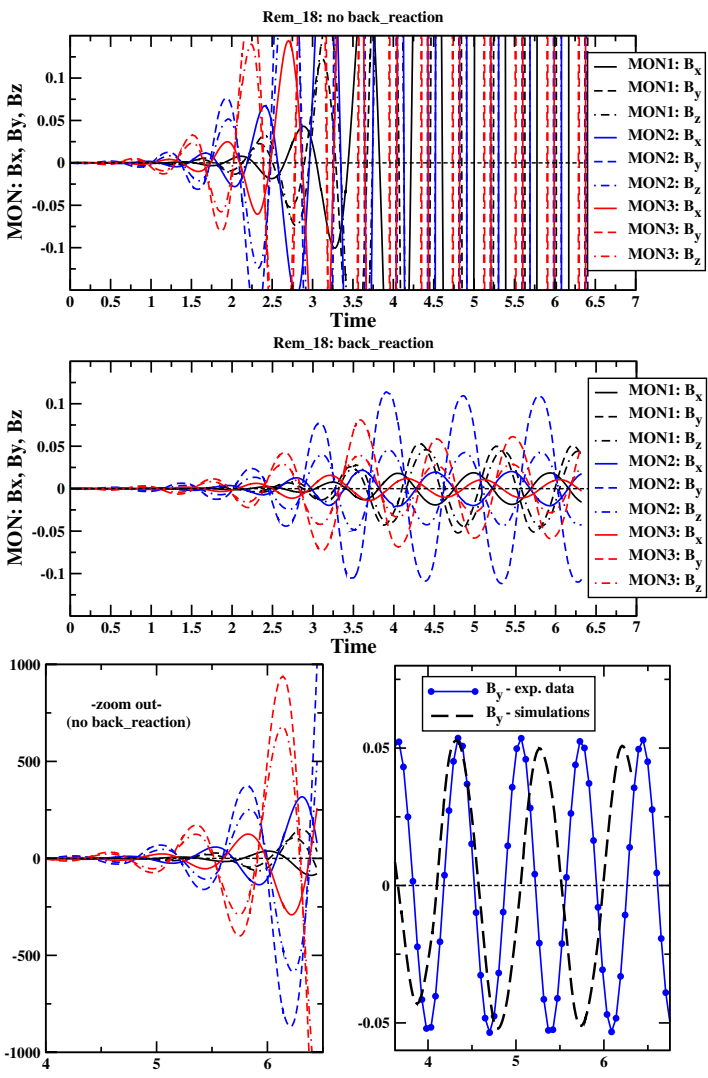

FIG. 3 (color online). Time evolution (in s) of magnetic field components (in $T$ ) at characteristic monitoring locations (MON1-MON3) without and with backreaction (two-way coupling) in the Riga-dynamo experiment, $\mathrm{Re}=3.5 \times 10^{6}, \mathrm{Re}_{m}=$ 18. The monitoring locations are defined at $\operatorname{MON} 1(0.16,1,0)$, $\operatorname{MON} 2(0.16,0,0),. \operatorname{MON} 3(0.16,-1 ., 0)$. The right figures are at the MON2 location (central part of the outer passage). 


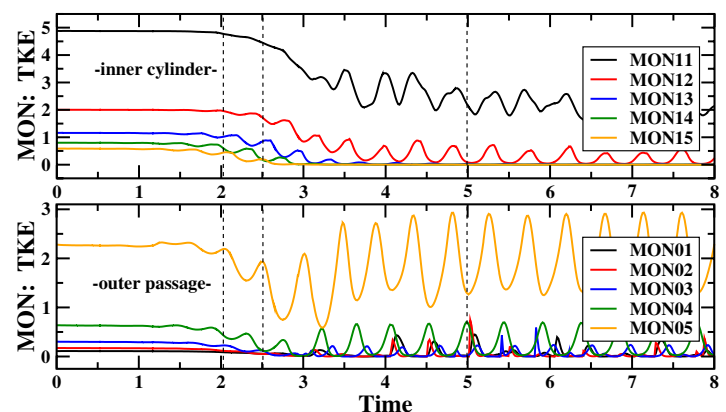

FIG. 4 (color online). Time evolution (in s) of the turbulent kinetic energy (in $\mathrm{m}^{2} / \mathrm{s}^{2}$ ) at the characteristic monitoring points distributed along the inner cylinder (MON01-MON05) and outer passage (MON11-MON15) - as sketched in Fig. 1.

force effect, and the initial values are clearly surpassed. It is interesting to observe periodic outbursts of TKE in the upper part of the outer passage with a double frequency of the recorded self-generated axial magnetic field at the same locations.

The dynamics of the spatial evolution of the created magnetic field is illustrated in Fig. 5. The magnetic flux lines (generated from the instantaneous magnetic field components) portray nicely the organized double-helix magnetic structures at the later growth stage. The isosurfaces (red and blue indicate identical values of the opposite poles) of the axial magnetic field are superimposed in order to identify regions or locations with the strongest selfamplification. The animations of the numerical results show slowly rotating asymmetric magnetic field oscillating in axial direction with a frequency of $\approx 1 \mathrm{~Hz}$ (compared with eigenfrequency of the driving propeller $\approx 30 \mathrm{~Hz}$ ). It is interesting to note that numerical simulation revealed ex-

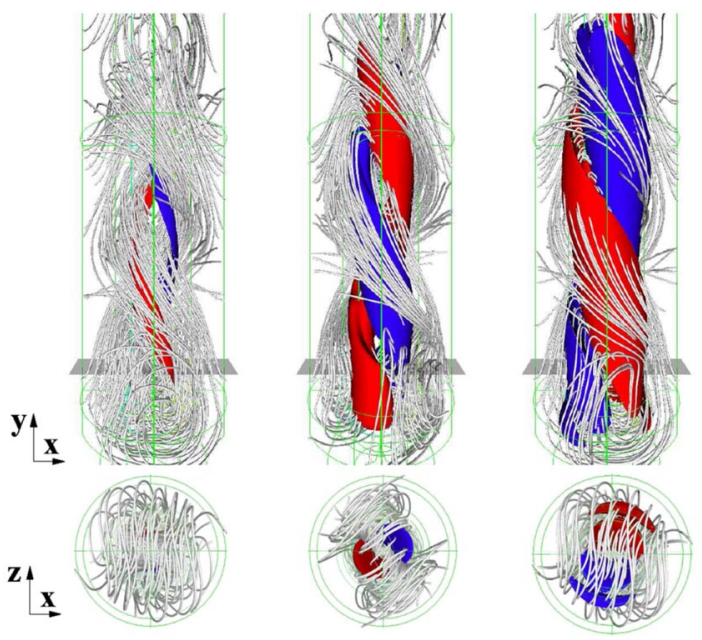

FIG. 5 (color online). Spatial distributions of growing magnetic field in the Riga-dynamo experiment: isosurfaces of the axial magnetic field component $\left(B_{y}=0.02-\right.$ dark $/$ red; $B_{y}=$ -0.02 (in $T$ ) - light/blue) with magnetic flux lines: above-a side view; below - a view from below. Time $=2,2.5$, and $5 \mathrm{~s}$, respectively — as marked in Fig. 4. perimentally observed mechanism of the upward shift of the magnetic field intensity when saturation regime is finally reached. This is a consequence of the flow reorganization due to simultaneous reduction of both the axial and tangential velocity components in the inner cylinder due to the self-generated Lorentz force. More detailed analysis of the flow and magnetic fields, their interactions and energy exchange, and of the kinematic and magnetic vortical structure and their interaction, is currently underway and will be reported in a subsequent publication.

This research was supported by EC project "MAGDYN" Contract No. HPRI-CT-2001-50027 and by the Royal Netherlands Academy of Arts and Sciences (KNAW). The supercomputing facilities were provided by the NWO/NCF. Dr. F. Stefani, Dr. G. Gerbeth, and Professor A. Gailitis are gratefully acknowledged for numerous discussions and for providing the experimental data.

[1] G. Rüdiger and R. Hollerbach, The Magnetic Universe: Geophysical and Astrophysical Dynamo Theory (Wiley, Weinheim, 2004).

[2] A. Gailitis et al., Phys. Rev. Lett. 84, 4365 (2000).

[3] A. Gailitis et al., Phys. Rev. Lett. 86, 3024 (2001).

[4] A. Gailitis et al., Rev. Mod. Phys. 74, 973 (2002).

[5] A. Gailitis et al., Surv. Geophys. 24, 247 (2003).

[6] A. Gailitis et al., Phys. Plasmas 11, 2838 (2004).

[7] R. Stieglitz and U. Müller, Phys. Fluids 13, 561 (2001).

[8] U. Müller, R. Stieglitz, and S. HoranyiJ. Fluid Mech. 498, 31 (2004).

[9] U. Müller and R. Stieglitz, Nonlin. Proc. Geophys. 9, 165 (2002).

[10] A. A. Schekochihin et al., Phys. Rev. Lett. 92, 054502 (2004).

[11] Y. Ponty, H. Politano, and J. F. Pinton, Phys. Rev. Lett. 92, 144503 (2004).

[12] Y. Ponty et al., Phys. Rev. Lett. 94, 164502 (2005).

[13] P. D. Mininni et al., Astrophys. J. 626, 853 (2005).

[14] G. A. Glatzmaier and P. H. Roberts, Nature (London) 377, 203 (1995).

[15] A. Yoshizawa, Hydrodynamic and Magnetohydrodynamic Turbulent Flows: Modelling and Statistical Theory (Kluwer, Dordrecht, 1998).

[16] S. Kenjereš and K. Hanjalić, International Journal of Heat and Fluid Flow 21, 329 (2000).

[17] S. Kenjereš, K. Hanjalić, and D. Bal, Phys. Fluids 16, 1229 (2004).

[18] F. Hamba, Phys. Plasmas 11, 5316 (2004).

[19] S. Kenjereš et al., Phys. Plasmas 13, 122308 (2006).

[20] K. Hanjalić and S. Kenjereš, Flow, Turbul. Combust. 66, 427 (2001).

[21] K. Hanjalić and S. Kenjereš, J. Turbul. 1, 008 (2000).

[22] D. V. Gaitonde and J. H. Miller, AIAA J. 43, 1064 (2005).

[23] S. Satake, T. Kunugi, and S. Smolentsev, Fusion Eng. Des. 61, 95 (2002).

[24] J.E. Borovsky and H. O. Funsten, J. Geophys. Res., [Space Phys.] 108, 1246 (2003).

[25] SARA Computing and Networking Services, www.sara.nl.

[26] A. Brandenburg et al., Astrophys. J. 446, 741 (1995). 